\title{
Optimized Non-Obstructive Particle Damping (NOPD) Treatment for Composite Honeycomb Structures
}

\author{
H. Panossian* \\ Pratt \& Whitney Rocketdyne, Canoga Park, CA 91303
} \begin{abstract}
an optimal NOPD treatment design configuration and verification with test data.
Nomenclature

$\ddot{x}=$ Acceleration

$\dot{x} \quad=$ Velocity

$\mathrm{X}=$ Displacement

$F \quad=$ Force

$\omega=$ Frequency

$v_{1} \quad=$ ith Design Variable

c $\quad=$ Viscous damping

$\mathrm{c}_{i} \quad=$ Coefficient of ith Design Variable

$\zeta=$ Critical damping ratio

$\sigma_{j} \quad=$ Stress in the $j^{\text {th }}$ element, Constraint Parameter for Optimization

$\lambda_{\mathrm{i}} \quad=$ ith Eigenvalue

$A \quad=$ Amplitude of Motion

$W \quad=$ Average dissipated power

$\mathrm{t} \quad=$ Time

$\mathrm{k} \quad=$ Stiffness

$E_{I}^{F S}, E_{2}^{F S}=$ Elastic modulus, superscript stands for face sheet and subscript for direction

$G_{13}{ }^{F S}, G_{23}{ }^{F S}=$ Shear modulus, superscript stands for face sheet and subscript for direction

$[M],[C],[K]=$ Mass, Damping and Stiffness matrices of the structure

$\alpha, \beta=$ Mass \& Stiffness related damping ratios
\end{abstract}

Abstract: Non-Obstructive Particle Damping (NOPD) technology is a passive vibration damping approach whereby metallic or non-metallic particles in spherical or irregular shapes, of heavy or light consistency, and even liquid particles are placed inside cavities or attached to structures by an appropriate means at strategic locations, to absorb vibration energy. The objective of the work described herein is the development of a design optimization procedure and discussion of test results for such a NOPD treatment on honeycomb (HC) composite structures, based on finite element modeling (FEM) analyses, optimization and tests. Modeling and predictions were performed and tests were carried out to correlate the test data with the FEM. The optimization procedure consisted of defining a global objective function, using finite difference methods, to determine the optimal values of the design variables through quadratic linear programming. The optimization process was carried out by targeting the highest dynamic displacements of several vibration modes of the structure and finding an optimal treatment configuration that will minimize them. An optimal design was thus derived and laboratory tests were conducted to evaluate its performance under different vibration environments. Three honeycomb composite beams, with Nomex core and aluminum face sheets, empty (untreated), uniformly treated with NOPD, and optimally treated with NOPD, according to the analytically predicted optimal design configuration, were tested in the laboratory. It is shown that the beam with optimal treatment has the lowest response amplitude. Described below are results of modal vibration tests and FEM analyses from predictions of the modal characteristics of honeycomb beams under zero, $50 \%$ uniform treatment and

\section{Introduction}

Research in the field of particle mechanics has shown the complexity of the response of an ensemble of granular particles, placed in a cavity in a vibrating structure, under dynamic excitation. At microscopic scales energy dissipation mechanisms in solids are poorly understood, since these depend on a large number of parameters. Thus, particle damping design tools for application on vibrating structures is not available $e^{1-3}$.

Particle mechanics has been investigated for several decades. The recent interest in the damping capability of particles in vibrating structures has given a boost to this area of research ${ }^{4}$. Numerous organizations and universities in many countries are currently experımentıng with particles for damping structural and acoustic vibrations as well

" PWR Tech Fellow, 6633 Canoga Ave.- AC56, Canoga Park, CA 91303. AIAA Ass. Tech Fellow 
as to study the various complex mechanisms of vibration/noise energy absorption in this robust technique. Being at least as effective as other damping techniques (e.g., viscoelastic damping), it is the only one, other than friction dampers, that is insensitive to harsh environmental conditions. NOPD is virtually unaffected by temperature, unlike viscoelastic damping ${ }^{5}$. Because NOPD can be integrated into existing hardware, it has also proven to be a technology that can be used to solve vibration problems in numerous fields ${ }^{5-7}$.

The particle-damping process involves friction and other energy dissipation mechanisms. Friction is the macroscopic physical manifestation of cohesive and adhesive surface forces between particles; so anything beyond semi-empirical laws becomes extremely complicated. The combination of elastic, viscous, and plastic mechanisms during collisions and deformations of the granular systems lead to meso-scopic properties that provides researchers a formidable topic for research. Theoretical advancements in this area are limited to simplified systems. There is a lot of recent parametric or experimental research on particle instabilities because of convection, size segregation, fingering, ripple and stripe formation, and other phenomena found in granular ensembles out of equilibrium ${ }^{8}$. Numerical simulation of the behavior of particles presents a new way of understanding interaction mechanisms and patterns of flow, energy dissipation, and dynamic regimes.

NOPD may be considered as a generalization of the well-known impact damper, in that the latter only uses a single particle or mass, while in the former, numerous particles are placed in the cavity ${ }^{9}$. Similar to impact dampers, particles sometimes move out of phase with the cavity walls, resulting in impacts and creating frictional forces amongst the particles and between the particles and the surrounding walls, and these provide the mechanisms for energy absorption ${ }^{10}$. The objective of the work described herein is the development of a design optimization procedure and test results for such a NOPD treatment on honeycomb composite structures, based on finite element modeling (FEM) analyses, optimization and tests. Modeling and predictions were performed and tests were carried out to correlate the test data with the FEM. The optimization procedure consists of defining a global function, using finite difference methods, to determine optimal values of the design variables through quadratic linear programming. The optimization of NOPD treatment design variables under structural dynamic loading is complicated due to the distribution of particles and consideration of responses of several modes for amplitude minimization. Most researchers have studied structural design optimization by taking minimum weight as objective function, under constraints on static displacements and stresses. However, little effort has been made for optimal design of dynamically constrained structures, such as this case. To prevent potential resonance between a structure and applied forces, designs are carried out such that the response amplitudes of selected modes are minimized under dynamic loading near the natural frequencies. Herein optimization was carried out on those selected frequencies within the structural response spectrum where high stresses can cause failure and where damping can be effective

A Standard Genetic Algorithm (SGA) was used in parallel with the Sequential Quadratic Programming (SQP) on a honeycomb beam structure. The SGA has a linear ranking scheme for parent selection, a set of basic genetic operators (crossover, mutation, and gene swap), and an elitist selection procedure. This approach provides points close to the globally optimal, but it is very computationally intensive and lacks robustness. However, to start the optimization process for the SQP algorithm these initial points are used, with the nonlinear optimization constraints, to determine the optimum. The SQP is solved for every initial point predicted by SGA. NOPD treatment optimization requires amount/weight minimization of particles subject to acceptable dynamic responses. The design variables are parametric and multivariable so that continuous spatial control of the NOPD can be maintained independent of mesh regiments and natural frequencies.

An optimal design was derived and laboratory tests were conducted to evaluate its performance under different vibration environments. Three honeycomb composite beams, with Nomex core and aluminum face sheets, empty (untreated), uniformly treated with NOPD, and optimally treated with NOPD, according to the analytically predicted optimal design configuration, were tested in the laboratory. The beams were suspended with rubber bungee chords and structurally excited by a hammer and/or shaker. It is shown that the beam with optimal treatment has the lowest response amplitude.

\section{II. $\square$ Finite Element Analysis \& Optimization}

The mechanics of solid particles and their damping or acoustic attenuation effectiveness in a structure involves complex relations and parameters ${ }^{6-10}$. To study the damping effectiveness of light particles, placed inside the cells of stiff honeycomb structures, a one year duration test and analysis program was initiated in Pratt \& Whitney Rocketdyne (PWR) in September 04 (Boeing, Rocketdyne Propulsion \& Power at that time). This program was sponsored by the NASA Langley Research Center (LRC), Structural Acoustics Branch. In this study laboratory vibration/modal testing and finite element analyses were carried out to predict the modal characteristics of the honeycomb beams, provided by NASA, and model-test correlations were performed to anchor the FEM with test 
data. These tests were then repeated under different NOPD treatments of the honeycomb to characterize the particle and fill configuration effects on performance. The beams were suspended with rubber bungee chords (free-free) and structurally excited by a shaker/hammer. Analysis was then carried out, using a correlated FEM, to find an optimized treatment profile in the frequency range of interest, by considering a number of predetermined parameters/constraints for the optimal NOPD treatment configuration.

Three beams of composite NOMEX core and aluminum face-sheet honeycomb, with dimensions of 46 in $\mathrm{x} 2$ in x 0.75 in were tested for modal parameter identification. The FEM was developed in ABAQUS software and used for predictions of these modal characteristics. Random excitations were initially used in the laboratory to determine the frequencies of the beam fundamental modes and their preliminary damping values. Sinusoidal shaker excitations and hammer inputs were then used to characterize damping of each individual mode. Optimization was carried out to determine the best NOPD treatment configuration, whereby target frequency responses, of specific modes, were minimized with an appropriate objective function.

In the FEM of the beam, the honeycomb cells were modeled as individual solid elements while the face sheets were modeled with separate solid elements. The model is solved in ABAQUS. Several analyses were performed to estimate the passive damping properties of the NOPD material based on test data obtained in vibration/modal tests. The face-sheets were assumed to be orthotropic. The HC core itself was modeled as an orthotropic material with perturbations in density and damping to represent the NOPD materials tested. For correlation with tests and determination of modal damping of a particular mode, frequency dependent modal composite damping was used. To predict total response direct integration was used with material damping and nonlinear dependence on frequencies. Several numerical analysis steps are required to estimate the passive broad-band damping properties of the NOPD material. Optimal correlation of the beam parameters, without NOPD treatment, was first performed to match the elastic properties and the baseline system damping with those of the lab tests. A Python computer program was written to automatically generate input files to ABAQUS, to run and post-process the results without user intervention. The Python code generates five types of linear finite element solutions: eigenvalue, harmonic, harmonic with composite damping, random, and harmonic direct integration with passive broad-band damping. The prediction of the responses and the analyses involved are nonlinear (linear dynamic perturbation theory), and was performed in Abaqus with the Python code and an optimization algorithm. The script was written such that refinement is achieved by changing a single parameter and studies were performed for all five types of analyses. The final mesh density of the FEM was determined by refinement studies and six degrees of freedom (DOF) were used to capture minimum wavelength in the structure. The glue plus the face-sheet was modeled as a single layer within a plane stress laminate structure, so the density and the dynamic modulus of the aluminum were macroscopically smeared. The face-sheet properties were assumed to be orthotropic lamina in plane stress, since it is symmetrical through its thickness. The HC was modeled similarly with perturbations in density and damping to represent the NOPD material. Out-of-plane warping due to non-symmetrical stiffness through the HC thickness was assumed negligible as compared to the face-sheet stiffness.

The approach taken in the NOPD treatment optimization was to minimize the damping treatment material weight, with constraints in the continuous design variables of the dynamic responses of the structure subject to nonlinear constraints on selected modes. Design variables were defined as piecewise continuous multivariate spline functions with evenly distributed control knots. To this end a Standard Genetic Algorithm (SGA) was used in conjunction with the Sequential Quadratic Programming (SQP) on a honeycomb beam structure. The SGA uses a linear ranking scheme for parent selection, a set of basic genetic operators (crossover, mutation, and gene swap), and an elitist selection procedure. This approach provides points close to the globally optimal, but it is computationally very intensive and lacks robustness. However, when these SGA points are used as initial values to start the optimization process for the $\mathrm{SQP}$ algorithm, with the nonlinear optimization constraints, it is possible to determine the local bounded minima and, thus reduce the targeted dynamic responses. Multiple initial points were extracted from the SGA for local SQP multimodal search spaces, where a distance metric was defined over the design variable search space. This process helped SQP to identify a unique search space. The SQP was solved for every initial point predicted by SGA to get the optimum.

\section{A. Mathematical Formulation of the Optimization Problem:}

In structural dynamic optimizaliun (SDO) problems, constraints on design variables, such as static response (e.g., static stress, displacement, local stability, and their time derivatives), dynamic response (e.g., frequency response and impulse response) and natural frequency are often used. Suppose the $i^{\text {th }}$ design variable is $v_{\mathrm{i}}$, which usually 
represents a set of elements having the same NOPD treatment, and $c_{i}$ as the coefficient corresponding to $v_{i}$. Moreover, let $\sigma_{\mathrm{j}}$ represent a set of dynamic response constraints on the $i^{\text {th }}$ eigenvalue and $\lambda_{\mathrm{i}}$ represent the prohibited domains of natural frequencies and specifications on particular natural frequencies. $S$ is a given set of available parametric function limits, which implicitly include the lower and upper bounds of design variables. The SDO problem can be written as:

$$
\begin{array}{cl}
\text { minimize } & W=W\left(v_{i}\right)=\sum_{i=1}^{k} c_{i} \dot{v}_{i} \\
\text { subject }- \text { to } & \sigma_{j} \leq \bar{\sigma}_{j} \Rightarrow u_{j} \leq \bar{u}_{j}, \dot{u}_{j} \leq \overline{\dot{u}}_{j}, \ddot{u}_{j} \leq \bar{u}_{j} \\
& a_{m n} \leq \bar{a}_{m n} \\
& g_{j}(v) \leq 0 \\
& \bar{\lambda}_{i}^{0}, \geq \lambda_{i} \leq \bar{\lambda}_{i}, \\
& v \in S(x, y)=\left(S_{j}: j=5 \%, \ldots, 95 \%\right)
\end{array}
$$

where: $(W)_{\mathrm{cyc}}=\mathrm{mbA}^{2} \omega^{2}, \sigma_{\mathrm{j}}$ is the stress in the $\mathrm{j}^{\text {th }}$ element, which implies proportional linear responses when subjected to linear dynamics, and $a_{m n}$ is the amplitude of the frequency response of the $\mathrm{m}^{\text {th }}$ degree of freedom excited at $\mathrm{n}^{\text {th }}$ degree of freedom, respectively. The bar over a variable represents the target value of the variable.

The structural forced vibration equation, based on the finite element method, can be formulated using the mass, damping and stiffness matrices of the structure as $[M],[C]$ and $[K]$, respectively. In the set of equations (3), $\ddot{x}, \dot{x}, x$ are acceleration, velocity and displacement vectors respectively, and $u$ is their relative deflections; $\{f\}$ is the external force vector.

$[M]\{\ddot{x}\}+[C]\{\dot{x}\}+[K]\{x\}=\{f\}$

$\{\ddot{x}\}+2 \varsigma_{i}\{\dot{x}\}+\omega_{i}^{2}\{x\}=\{f(\Omega)\}$

$[K]\left\{\phi_{i}\right\}=\lambda_{i}[M]\left\{\phi_{i}\right\}$

where $\left\{\phi_{i}\right\}$ is the eigenvector corresponding to the eingenvalue $\lambda_{i}$. The amplitude of the frequency response can then be obtained as

$$
\begin{aligned}
& a_{m n}=\sqrt{\mathrm{Re}_{m n}^{2}+\operatorname{Im}_{m n}^{2}} \\
& \operatorname{Re}_{m n}=\phi_{m i} \phi_{i n} \frac{\lambda_{i}-\Omega^{2}}{\left(\lambda_{i}-\Omega^{2}\right)^{2}+4 \zeta_{i}^{2} \lambda_{i} \Omega^{2}}, \quad \operatorname{Im}_{m n}=\phi_{m i} \phi_{i n} \frac{2 \zeta_{i} \lambda_{i}^{1 / 2} \Omega^{2}}{\left(\lambda_{i}-\Omega^{2}\right)^{2}+4 \zeta_{i}^{2} \lambda_{i} \Omega^{2}}
\end{aligned}
$$

in which $\Omega$ is the external frequency, $n$ is the number of degrees of freedom of the structure and $\zeta_{i}$ is the $i^{\text {th }}$ modal damping ratio. To SDO problem is obtained in two steps. First, a feasible solution is sought which satisfies all of the given constraints, using continuously changeable design variables. Then the optimum solution is determined based on the predicted set of feasible solutions.

\section{B. Feasibility Space:}

In the optimization process, several constraints may become simultaneously critical. The characteristics of frequency responses for various natural frequencies are, in general, different. To improve the efficiency in constraint satisfaction, we define a normalized constraint function, as

$Z(v)=\frac{\left|a_{m n}-\bar{a}_{m n}\right|+a_{m n}-\bar{a}_{m n}}{2 \bar{a}_{m n}}+\frac{\left|\lambda_{i}-\bar{\lambda}_{i}\right|+\lambda_{i}-\bar{\lambda}_{i}}{2 \bar{\lambda}_{i}}+\frac{\left|\lambda_{i+1}-\bar{\lambda}_{i+1}\right|+\lambda_{i+1}-\bar{\lambda}_{i=1}}{2 \bar{\lambda}_{i+1}}$

A necessary and sufficient condition for a solution to the SDO problem is to satisfy the constraints $Z(U)=0$. Using this constraint function, the optimization problem can be written as 
minimize $\quad W=W\left(v_{i}\right)=\sum_{i=1}^{k} c_{i} v_{i}$

subject to $Z\left(\nu_{i}\right)=0$

$$
\begin{aligned}
& \ddot{u}_{j} \leq \overline{\ddot{u}}_{j} \\
& v_{i} \in S=\left(s_{j}: j=1,2, \ldots . . N\right)
\end{aligned}
$$

Generally, the solution is obtained in an iterative manner. The structural dynamic characteristics are calculated in each cycle to find the incremental changes and to determine whether the constraints are satisfied or not. For a large or complicated structure with a large number of degrees of freedom approximation methods are usually used to improve the computational efficiency.

The structural eigenvalue problem can be formulated as:

$$
\left(([K]+[\Delta K])-\lambda_{i}^{m}([M]+[\Delta M])\right)\left\{\phi_{i}^{m}\right\}=0
$$

in which $[\Delta K]$ and $[\Delta M]$ are the incremental changes in the stiffness and mass matrices due to the structural modification in each iterative solution, respectively, $\lambda_{i}^{m}$ is the $i^{\text {th }}$ eigenvalue of the structure and $\left\{\phi_{i}^{m}\right\}$ is its corresponding eigenvector. Let $\left\{\phi_{i}^{m}\right\}=\left\{\phi_{i}\right\} q_{i}=\{\bar{\phi}\}\{q\}$ so that

$$
\left(\left[K_{0}\right]+[\bar{\phi}]^{T}[\Delta K][\bar{\phi}]\right)-\lambda_{i}^{m}\left([I]+[\bar{\phi}]^{T}[\Delta M][\bar{\phi}]\right)\left\{q_{i}\right\}=0
$$

in which $K_{0}$ is a diagonal matrix with $m_{1}$ eigenvalues, with a much lower dimension than the original one. For the NOPD honeycomb elements $\Delta K$ was assumed to be very small and only the original stiffness of the structure has been retained. By finding a feasible solution which satisfies the response and natural frequency constraints, the optimum values were automatically derived.

Damping was determined as a modal component, $\zeta_{i}$, and was taken as mode shape dependent for every, $\phi_{i}$, eigen vector. The damping parameter in the analysis was assumed independent of frequency. The finite elements have continuous damping distributions, for both treated and untreated honeycomb beams. This procedure is practical and efficient and it can deal with not only constraints of stress, displacement, and their derivatives, but also frequency response.

In the analysis a piece-wise Hermite geometric form bi-cubic polynomial was used as the NOPD distribution function, to represent the treatment parameter used for the optimization of the design variables. The bi-cubic Hermite mapping basis of mono-variate polynomials forms smooth blending functions that force second order continuity across boundaries. These mappings are applied, element by element, on $4 \times 4$ local arrays of points and vectors, arranged in the standard way, two extreme points with their derivatives at these extreme points. Design variables, therefore, consist of both the extreme points and their derivatives and control the NOPD damping mesh independently.

The mass of the particles as well as their induced damping effects shifts the frequency peak responses to lower values. For this reason a forced superposition harmonic sweep for a given frequency range was required for every mode. The peak response amplitude, $\boldsymbol{a}_{m n}$, was used as the nonlinear constraint during a sine sweep around the peak. All the responses were evaluated at every design point. As the number of design variables are increased the fidelity of the NOPD treatment distribution becomes less and less smooth and all the damping treatment approaches a discreet solution at three points (Figure 1. location A). However the physical limitation placed on the fill characteristics (Figure 1 location C) forced the optimizer to spread out the treatment (Figure 1 location B). The latter is considered as the optimal fill configuration for the honeycomb beam, considering the practicality issues of filling the honeycomb with particles. 


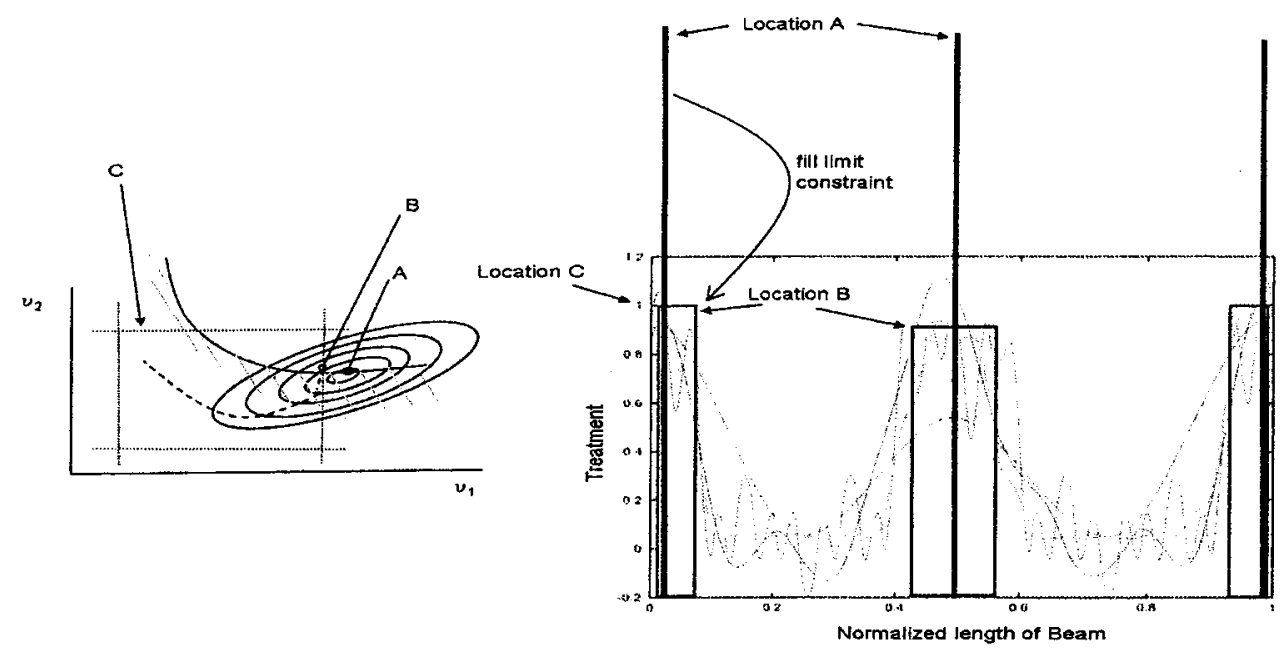

Figure 1. Sketch of the NOPD optimal treatment

\section{Derivation of Optimal Treatment Configuration}

A composite $\mathrm{HC}$ beam is investigated for optimal NOPD treatment. The added mass of the particles was taken as a performance criterion, with constraints on the response amplitudes (of a few target modes) and the mass. A finite element structural model was used to optimally determine both the placement of particles and parameters describing associated modal components in a damped structure. The technique utilizes a mechanical theory to analytically determine the response of arbitrary structures with a damping material. The optimal solution uses a fully coupled theory requiring simultaneous models of both the structural and the damping/material components, and the complex state of superposition of forces that may exist due to the particles, thereby providing the mechanical response reductions. A robust optimization procedure was developed to design the NOPD system for a potential of simultaneous damping of several critical modes of interest and was simplified by assuming linear modal superposition.

1. Simplex Method: In 1962 an efficient sequential optimization method called the basic simplex method was presented by Spendley et $\mathrm{a}^{16}$. This method will find the true optimum of a response with fewer trials than the nonsystematic approaches or the one-variable-at-a-time method. Two simplex algorithms are used simultaneously in the in the MultiSimplex optimization algorithms developed in order to enhance the capabilities of the simlex method. After the initial trials the simplex process is sequential, with the addition and evaluation of one new trial at a time. The simplex searches systematically for the best levels of the control variables. The optimization process ends when the optimization objective is reached or when the response reduction cannot be improved further.

The objective function attributed to minimizing the beam weight was defined in the form of a smooth function of the summation of all the individual masses of the added particles in each cell. However, the dynamic response constraint functions are inherently non-smooth. The nonlinear boundary constraints of the optimization were simulated as an overweight gradient in the objective function. The simplex algorithms can handle only one response at a time, but usually there are many response variables to optimize simultaneously. A method to form a joint response measure, from the individual response variables, was therefore needed.

2. Nonlinear Quadratic: Sequential Quadratic Programming (SQP) methods represent the state of the art in nonlinear programming methods. New advances in this approach allow mimicking Newton's method for constrained optimization just as is done for unconstrained optimization. Using a quasi-Newton updating method the Hessian of the Lagrangian function is approximated iteratively. This is then used to generate a SQP sub-problem whose solution is used to form a search direction for a line search procedure. It is assumed that bound constraints have been expressed as inequality constraints and then the quadratic programming sub-problem is created by linearizing the nonlinear constraints. A nonlinearly constrained problem can often be solved in less number of iterations than an unconstrained problem using SQP. One of the reasons for this is that, because of limits on the feasible region, the 
optimizer can make informed decisions regarding directions of search and step length.

A nonlinear optimization was also performed using Line-search Newton Conjugate Gradient, defined by quasianalytic response from Abaqus DSA and finite central difference defined by the optimizer itself. The low frequency modes were not as sensitive to material properties as high frequency modes, so the former were correlated first and then higher frequency modes were correlated. When used for the NOPD treatment optimization the solution worked for a limited set of design variables.

3. Nonlinear Genetic: Optimization difficulties arise when the equations in a model are not globally concave. This is often the case with nonlinear system models. The Genetic optimization algorithm combines evolutionary methods with a quasi-Newton approach. Appropriate formulation of the problem in an evolutionary manner can make the algorithm converge to the global optimum much more quickly. An evolutionary algorithm (EA) uses a collection of heuristic rules to modify a population of trial solutions in such a way that each generation of trial values tends to be on average better than its predecessor. The EA is fundamentally a genetic algorithm (GA) in which the code-strings are vectors of floating point numbers rather than bit strings, and the GA operators take special forms tuned for the floating-point vector representation. A GA uses a set of randomized genetic operators to evolve a finite population of finite code-strings over a series of generations The operators used in GA implementations vary, but in an analytical sense the basic set of operators can be defined as reproduction, mutation, crossover and inversion. Used in suitable combinations, the genetic operators tend to improve the average fitness of each successive generation ${ }^{17}$.

Due to the harshness of our highly nonlinear response boundary constrains, the genetic algorithm was to accommodate the large number of design variables required and increase the number of response constrains that define the nonlinear boundary conditions of the optimization. Local minima were first identified by suggestive quadratic methods from several local best breeding parent groups. The breeding produced more direct optimal offsprings and identified the local minima very quickly. The Annealing method using a local parent population was ideal for this situation.

It is critical to start with a large random population of initial points that can breed (and may have) a large genetic pool with enough gene diversity to identify the global minimum of several generations. Starting with a very diverse genetic population and accepting only the best to breed has a much faster convergence rate and a better chance of finding the global minimum, than letting genetic mutation drift take place over many-many generations.

4. Analytical Procedure: The first step is to model the beam HC structure as a composite laminate flat shell. Correlation of the bare beam characteristics (without NOPD passive damping) with test data is performed next to match the elastic properties and the baseline system damping of the beam from the lab test. The beam was modeled in Abaqus and matched to the dynamic test data, as closely as possible, by its dynamic response. Again, any out-ofplane warping due to non-symmetrical stiffness through the thickness of the $\mathrm{HC}$ was assumed negligible as compared to the stiffness of the face-sheets. The NOPD treatment was modeled as a new shell layer in the HC so that an element by element material damping could be controlled easily by composite damping. No parameters had to be changed in the Python code for different finite element model configurations. Results were automatically found and evaluated by the Python code as input to the next model for multiple restarts and optimization requirements. The response amplitudes of three modes were used, selected from a large range of the beam modes, for the optimization. The modes were selected from those with the highest response, from both test and analysis. The response amplitude was represented by the maximum FRF value, determined from several samplings near the undamped mode FRF, to make sure that the peak response point was found.

Optimization Results: Dynamic analyses were carried out on the steady-state amplitude and phase of the responses due to harmonic excitation at each frequency via a frequency sweep, similar to those in sine-sweep laboratory tests. By applying the loading at the same location as the test and normalizing the responses with respect to the load results comparable with the test were obtained. The response of interest, which was used for test-analysis correlation, is the normalized acceleration $[\mathrm{g} / \mathrm{lb}]$ at a corner of the beam.

The damping at selected frequencies was analytically determined by running many FEM cases with different damping values until the amplitude attenuation of the modes of interest were correlated with the test data. The critical damping ratios were calculated at the few selected dominant modes of interest and were then applied to the surrounding modes.

Figure 2 shows comparisons of the optimization results of the (quadratic combined with the genetic) optimizers under dliferent number or design varables. Here the highest weight is given in red, where the corresponding peak response occur, and these are minimized for three selected modes of interest. The quadratic optimizer praduced a lower weight but failed to make all responses below the targeted threshold $(1 \mathrm{~g} / \mathrm{lb})$ for one of the responses. The 
genetic algorithm added a little more weight but did produce the acceptable responses. Figure (3) shows the way the results were implemented for ease of application and test.

Optimization Resuls Nomalized Fin Levets
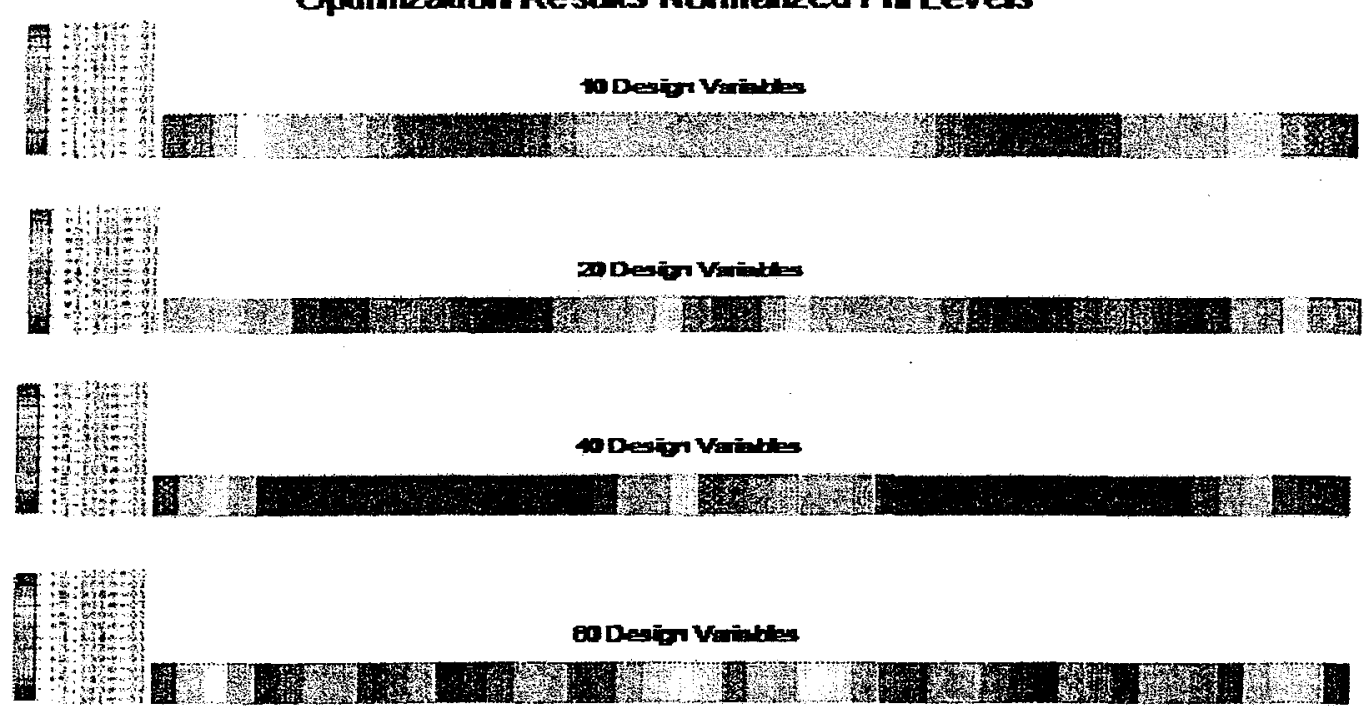

Figure 2. Optimal Predicted NOPD Fill Beam (Red=100\%, Green 50\%\& Blue 0\%)
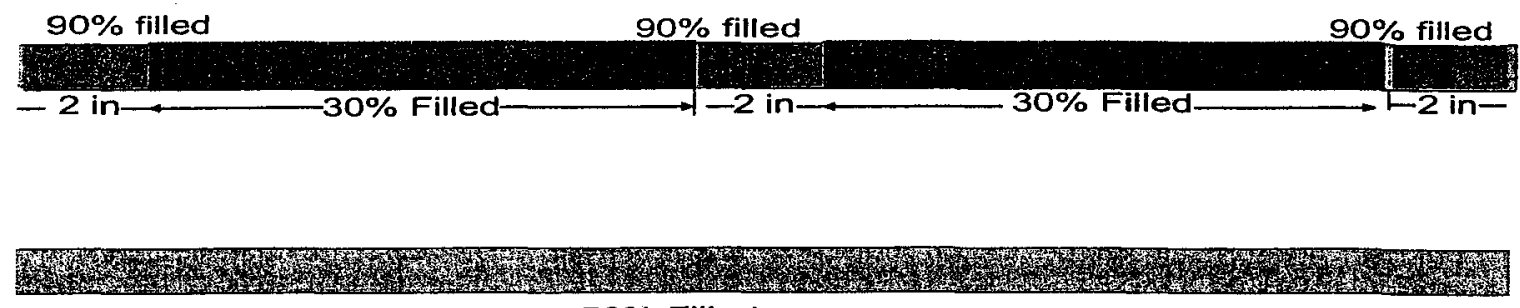

$50 \%$ Filled

O\% Filled- Empty

Figure 3. Optimal Implemented NOPD Fill Beam (Red $=100 \%$, Green 50\%\& Blue 0\%) (Weight of Empty Beam $=265 \mathrm{~g}$, Weight $50 \%$ Uniform $=285 \mathrm{~g}$ and OPT Genetic $=277 \mathrm{~g}$ )

D. Modal/Vibration Tests: Modal and vibration tests were carried out in the Engineering Development Lab (EDL) of PWR, to characterize the modal parameters of the honeycomb beams. Three beams with Nomex core and aluminum face sheets were used, one beam was unfilled and used for baseline purposes and a second beam was filled uniformly with Perlite particles $50 \%$ full, and the third one was filled according to the optimum configuration that was derived (Figure 3). They were tested free-free under identical suspension and vibration conditions for comparison. Sinusoidal and random shaker input tests were conducted to get a good estimate of modal damping. The results indicate the first bending mode is at $103 \mathrm{~Hz}$ for the undamped beam and at $101 \mathrm{~Hz}$ and $\sim 98 \mathrm{~Hz}$ for the beam with particles. The estimated damping value for the undamped beam is about $0.08 \%$ for the first bending mode, while for the $50 \%$ filled beam it was about $1.7 \%$ and for the optimum it was $\sim 2 \%$, respectively. Damping values for the second bending mode were $0.17 \%, 1.8 \%$ and $2.6 \%$ at $258 \mathrm{~Hz}, 256$ and $254 \mathrm{~Hz}$ frequencies for the three beams, respectively(see Figure 4). 

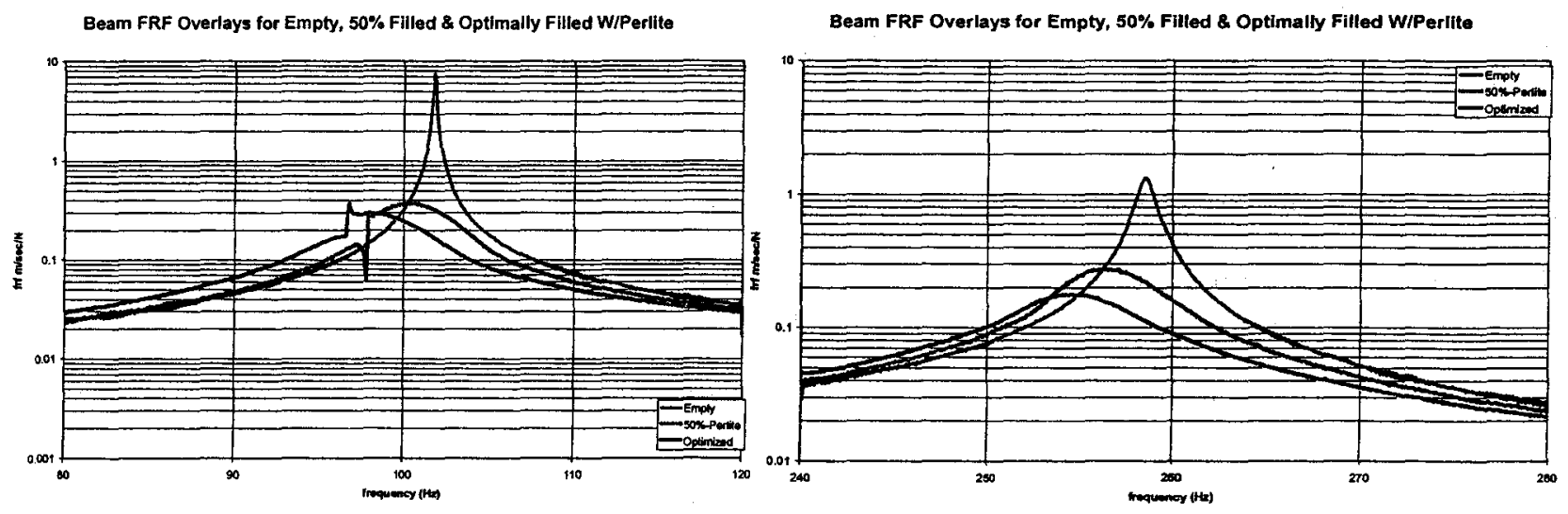

Beam FRF Overlays for Empty, 50\% Filled \& Optimally Filled W/Pertite

Beam FRF Overlays for Emply, 50\% Filled \& Optimally Filled WiPerlite
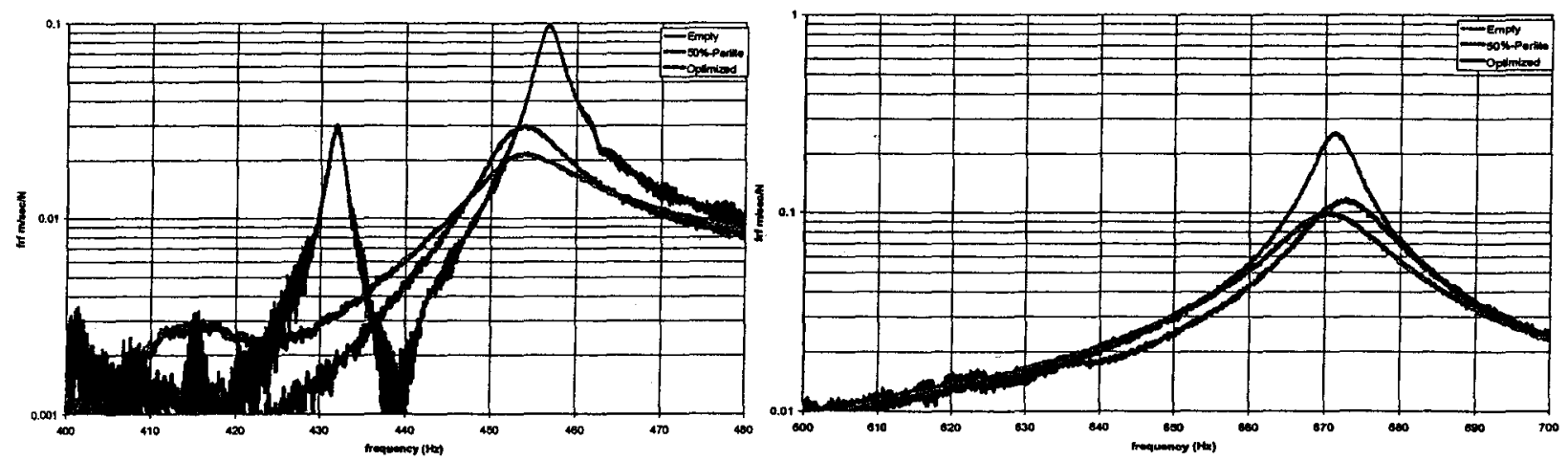

Figure 4. Beam Response FRF with \& W/O NOPD, Hammer Inputs

\section{III. $\square$ Conclusion}

The optimal configuration for NOPD treatment was derived through the use of ABAQUS FEM and a genetic optimization algorithm. The results indicate a treatment configuration that makes intuitive sense and tests indicate that the optimally treated beam performance was superior to the nominal/uniform fill configuration.

\section{Acknowledgments}

This program is sponsored by NASA Langley Research Center, in Hampton, Virginia, and the technical lead was Dr. Dan Palumbo.

\section{References}

${ }^{1}$ Saluena, C., Esipov, S.E., Rosenkranz, D. and Panossian, H.V.,"Modeling of arrays of passive granular dampers “, SPIE Proceedings 3672, Passive Damping and Isolation,1999, pp.32-42..

${ }^{2}$ Saeki, M., "Impact damping with granular materials in a horizontally vibrating system “, J. of Sound and Vibration, Vol. 251, No.1, March 2002.pp. 153-161.

${ }^{3}$ Fowler, B.L., Flint, E. and Olson, S.E., "Effectiveness and predictability of particle damping", SPIE Proceedings 3989, Damping and Isolation, 2000, pp.356-367. 
${ }^{4}$ Saluena, C., Esipov, S.E.,Poeschel,T. and Simonian S.S.," Dissipative properties of granular ensembles “, SPIE Proceedings 3327, Passive Damping and Isolation,1998, pp.23-29.

${ }^{5} \mathrm{H}$. Panossian, "Non-Obstructive Particle Damping Experience and Capabilities", Presented in the IMAC XX Conference, Feb 4-7,02 LA, CA

${ }^{6} \mathrm{H}$. Panossian, B. Kovac \& R. Rackl, "Composite Honeycomb Treatment Via Non-Obstructive Particle Damping (NOPD)", Presented in the SDM 04 Conference, April 19-22, 04 Palm Springs, CA.

${ }^{7}$ H. Panossian \& B. Kovac, "Optimal Non-Obstructive Particle Damping (NOPD) Treatment Configuration" ,Presented in the SDM 05 Conference, April 18-21, 05 Austin, TX.

${ }^{8}$ Powders \& Grains 97, Proceedings of the Third International Conference on Powders and Grains, edited by R. P. Behringer and J. T. Jenkins, Balkema, Rotterdam (1997).

${ }^{9}$ S. E. Semergil, N. Popelwell, and R. Tye, "Impact Damping of Random Vibration," J. Of Sound \& Vibration, V.121, pp 128-184 (1988).

${ }^{10}$ C. Salueña, S. E. Esipov, D. Rosenkranz, and H. V. Panossian, "On Modeling of Arrays of Passive Granular Dampers," in Proceedings of SPIE Vol. 3672, Smart Structures and Materials, Passive Damping and Isolation (1999).

${ }^{11} \mathrm{H}$. V. Panossian, "Non-Obstructive Impact Damping Applications for Cryogenic Environments," in Proceedings of Damping ' 89 Conference, Orlando, FL, pp. KBC-1, KBC-9. (February 1989).

${ }^{12}$ S. S. Simonian, "Particle Beam Damper," in Proceedings of SPIE Vol. 3672, Smart Structures and Materials, Passive Damping and Isolation (1999).

${ }^{13} \mathrm{H}$. V. Panossian, "Non-Obstructive Particle Damping Tests on Aluminum Beams," in the proceedings, Damping '91 Conference in San Diego, CA, pp. ICB-1, ICV-15, 13-15 (1991).

${ }^{14}$ H. V. Panossian, "Structural Damping Enhancement via NOPD Technique," in the Journal of Vibration and Acoustics, pp. 101-105, Vol. 114 (January 1992).

${ }^{15}$ H. V. Panossian, "An Overview of Non-Obstructive Particle Damping: A New Passive Damping Technique," in Shock and Vibration Technology Review, Vol. 1, No. 6, pp. 4-10 (1991).

${ }^{16}$ Spendley, W., Hext, G. R., Himsworth, F. R. "Sequential application of simplex designs in optimisation and evolutionary operation. Technometrics" 4(1962):4 441-461.

${ }^{17}$ Grefenstette, John J., and James E. Baker. 1989. "How Genetic Algorithms Work: A Critical Look at Implicit Parallelism." Proceedings of the Third International Conference on Genetic Algorithms, pp. 20-27. San Mateo, CA: Morgan Kaufmann. 